\title{
Miasis Uterina
}

\section{INVESTIGACION PATOLOGICA, CLINICA Y BIBLIOGRAFICA, LO MISMO QUE LA TOMA DE FOTOGRAFIAS REALIZADA POR EL DR. MIGUEL ANGEL ALARCON NIVIA*}

\section{SEGUNDO CASO EN COLOMBIA}

Miguel Angel Alarcón Nivia, Residente de Ginecobstetricia, realizador del trabajo.

Alberto Pérez, Jefe del Servicio de Sépticas, Hospital Ramón González Valencia. Bucaramanga.

\section{RESUMEN}

Se presenta el primer caso en Colombia de miasis uterina por Dermatobia hominis (Nuche) en una mujer campesina de 48 años, con prolapso genital total de un año de evolución. Hay un caso previo de miasis uterina por Callitroga americana (1) y otro de miasis vaginal por Dermatobia hominis (2). La literatura es muy escasa a nivel mundial.

\section{INTRODUCCION}

Las miasis son afecciones causadas por la invasión de larvas de moscas a tejidos $\mathbf{u}$ órganos del hombre o animales (3-8).

Entre las moscas de importancia médica se encuentran la Dermatobia hominis, que pertenece a la familia Cuterebridae, y la Callitroga americana que pertenece a la familia Calliphoridae (4).

Las moscas del género dermatobia tienen una fase larvaria obligatoria en los animales principalmente mamíferos y algunas aves (4), y en el hom-

Residente de Ginecobstetricia Universidad Industrial de Santander. Hospital Ramón González Valencia, Bucaramanga - Norte de Santander.

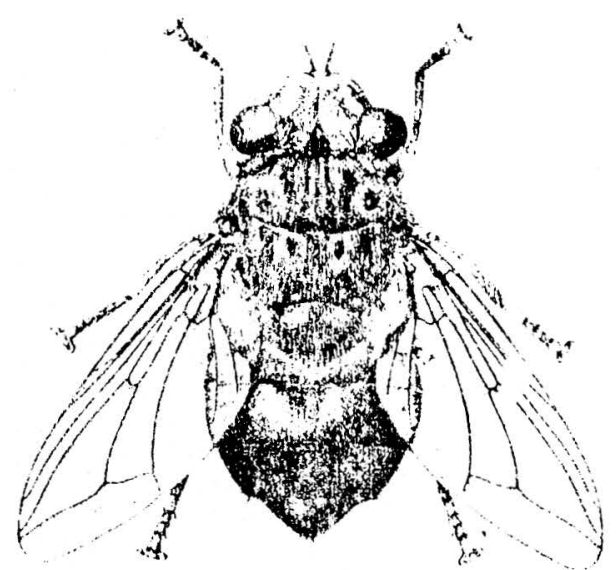

Figura 1. Dermatobia hominis

bre. La mayoría de las moscas productoras de miasis depositan ellas mismas las larvas en el huésped (4) pero la Dermatobia hominis tiene otra forma interesante de hacerlo: captura insectos hematófagos (principalmente mosquitos), les adhiere 15 a 30 huevos en un extremo del cuerpo mediante un pegante de secado rápido de tal manera que el polo por el que saldrá la larva apunta hacia atrás. Cuando el insecto se posa para chupar la sangre, el calor del nuevo huésped sirve de estímulo para que las larvas taladren la membrana del huevo y emigren por la piel, para lo cual aprovechan la herida producida por el mosquito. Guimaraes y Papavero (1966) describen 48 especies de mosquitos y moscas y una especie de garrapata que son utilizados como portadores (5). La Dermatobia hominis es común en Latinoamérica; mide 12 a $16 \mathrm{~mm}$, su cabeza es amarilla, su extremo posterior azul. Su forma exterior es parecida a una abeja. Sus larvas miden 18 a $24 \mathrm{~mm}$ $\mathrm{y}$ tienen aspecto vermiforme (6). Luego de 45 a 75 
días sale de la piel y se convierte en pupa la cual se convierte en mosca adulta 2 ó 3 semanas después (7).

Algunos géneros de moscas prefieren las cavidades corporales (nasal, urogenital, auricular, etc.), otros prefieren la piel, heridas, intestinos (4).

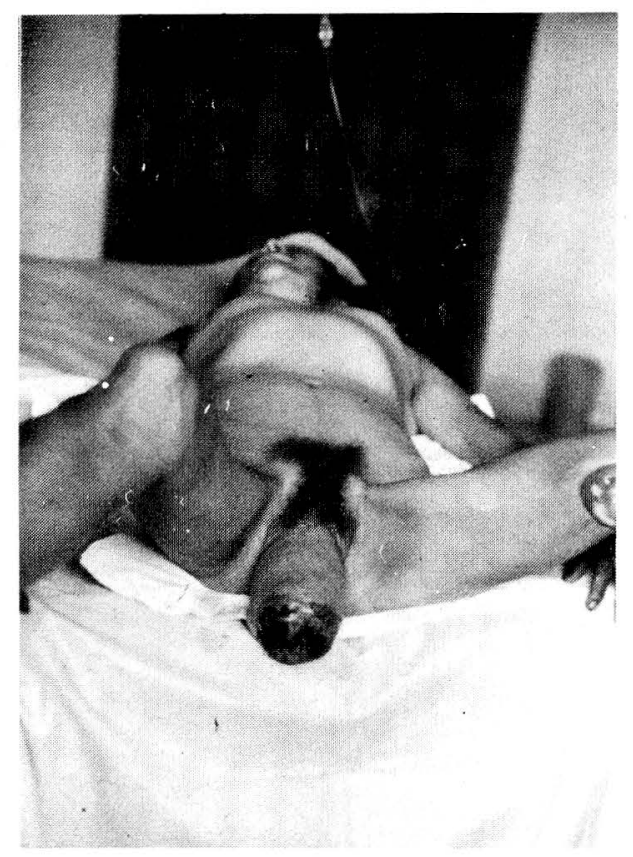

\section{RESUMEN DE HIS TORIA CLINICA}

H.C. No. 328135 Hospital Ramón González Valencia, Bucaramanga.

Edad: 48 años.

\section{Estado Civil: Casada}

Natural y procedente del área rural de San José de Suaita, Santander.

\section{Ocupación: Agricultura.}

Fecha de Ingreso: 27 de julio de 1987.

Mc.: masa genital y sangrado escaso persistente y fétido proveniente del útero desde hacía 2 meses. El prolapso genital era de un año de evolución. Refería poco aseo genital y la imposibilidad de usar ropa interior por el prolapso.

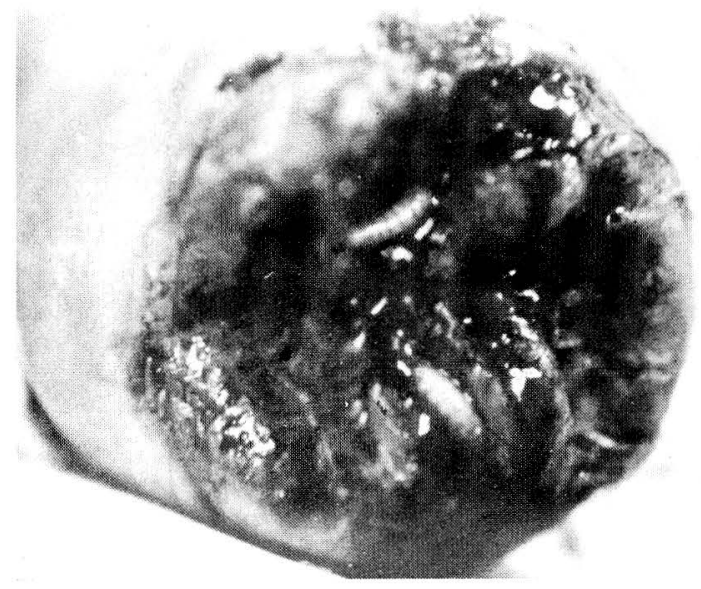

Antecedentes: hospitalizada 10 años antes por anemia al parecer por poliparasitismo en Vélez, Santander. G5P5 A.

Fup: 7 años antes. Todos los partos atendidos por parteras en su casa.

Examen de Ingreso: paciente desnutrida, pálida, su aspecto era de mayor edad que la real. Se apreciaba prolapso genital total con la presencia de múltiples túneles en el cuello por entre los cuales asomaban numerosos gusanos. Había secreción sero sanguinolenta muy fétida y constante

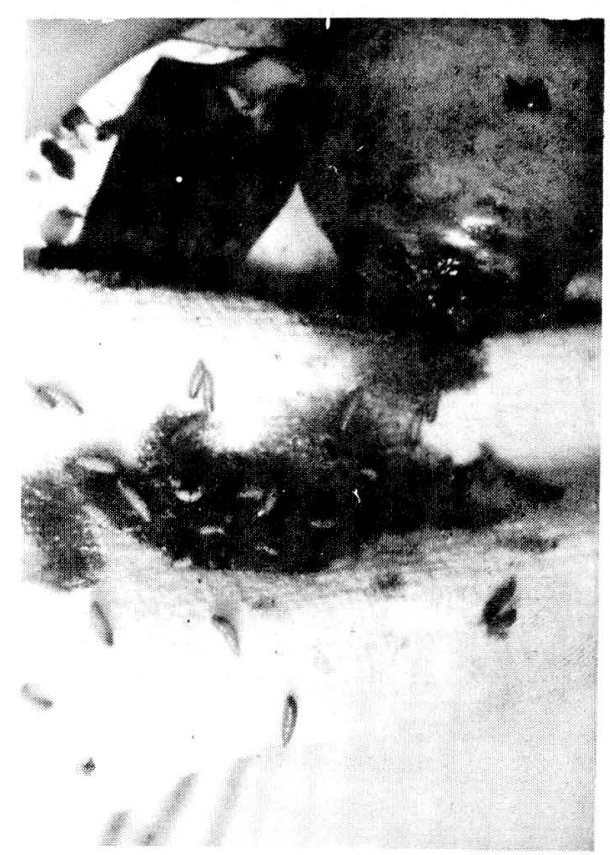




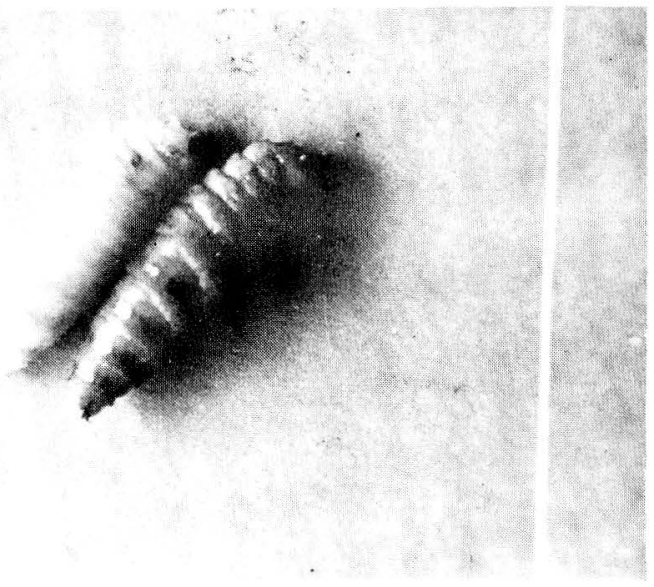

Tratamiento: Inicialmente se le extrajo la mayor parte de las larvas con pinza. Durante su hospitalización se completó la extracción con creolina, se le tomó biopsia del cervix que mostró cambios inflamatorios y se le tomaron pruebas de laboratorio pre-quirúrgicos. El coprológico mostró ascaris 20.000 por gramo de materia fecal, tricocéfalos $12.000 \mathrm{xg}$ MF y uncinarias $15.000 \mathrm{xg} \mathrm{MF}$. El cuadro hemático mostró moderada anemia. Una vez corregida la anemia, el parasitismo y estabilizada la

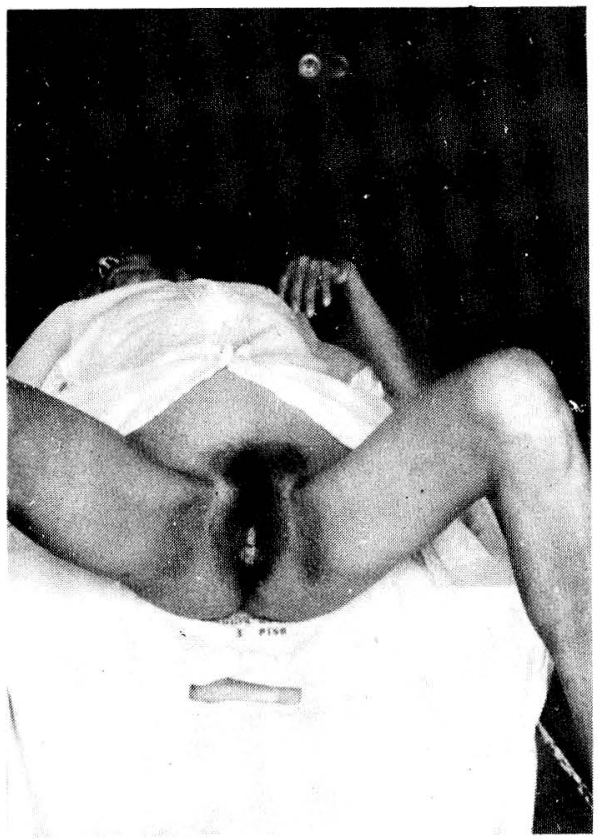

Después de varios días de hospitalización se le han extraído todos los gusanos y el útero ha sido introducido a la cavidad pélvica. paciente se le practicó histerectomía el 20 de agosto/87, cuyo reporte de patología dice: útero sir anexos que pesa 175 y mide $9.5 \times 8 \times 6 \mathrm{~cm}$. El cuello uterino irregular de múltipara mide $3 \mathrm{~cm}$ de diámetro transverso con múltiples desgarros bilaterales y moderado engrosamiento de epitelio exocervical. El endometrio suculento tiene un espesor de $7 \mathrm{~mm}$. El miometrio sin cambios histopatológicos específicos. No hay malignidad en los cortes examinados.

Diagnósticos: útero, histerectomía vaginal, cervicitis crónica severa activa con epidermización exocervical y metaplasia escamosa endocervical.

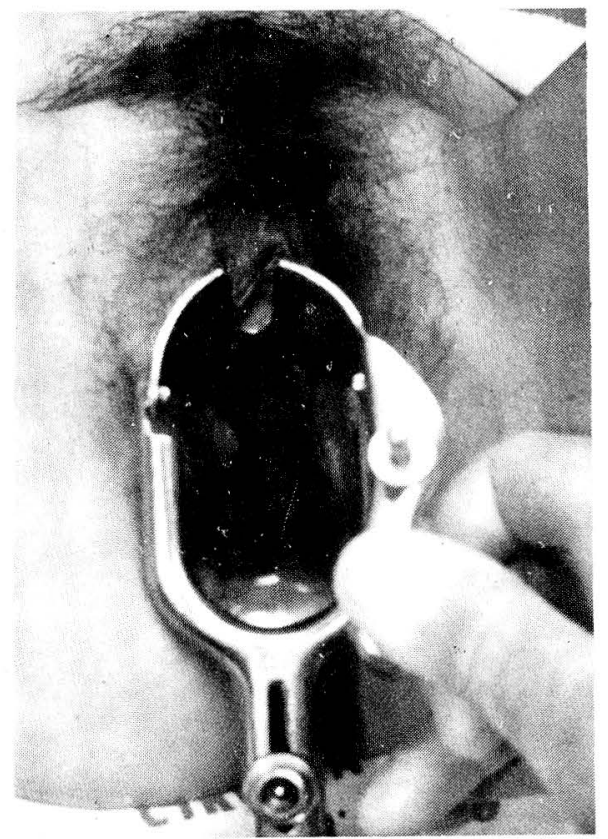

El cervix está en su sitio normal

\section{Endometrio Uterínico} cíficos.

Miometrio sin cambios histo-patológicos espe-

La evolución de la paciente fue satisfactoria por lo cual se le dio salida a final de agosto. Se llevaron las larvas al departamento de parasitología donde se intentó completar el desarrollo de las mismas pero no se logró obtener moscas adultas aunque sí se identificaron plenamente.

Se hizo búsqueda por computador por el sistema medlars-medline de la National Library of Bethesda, U.S.A. 
También se hizo búsqueda en la Index Medicux Latino Americano sin encontrar informes similares al actual en los últimos 28 años.

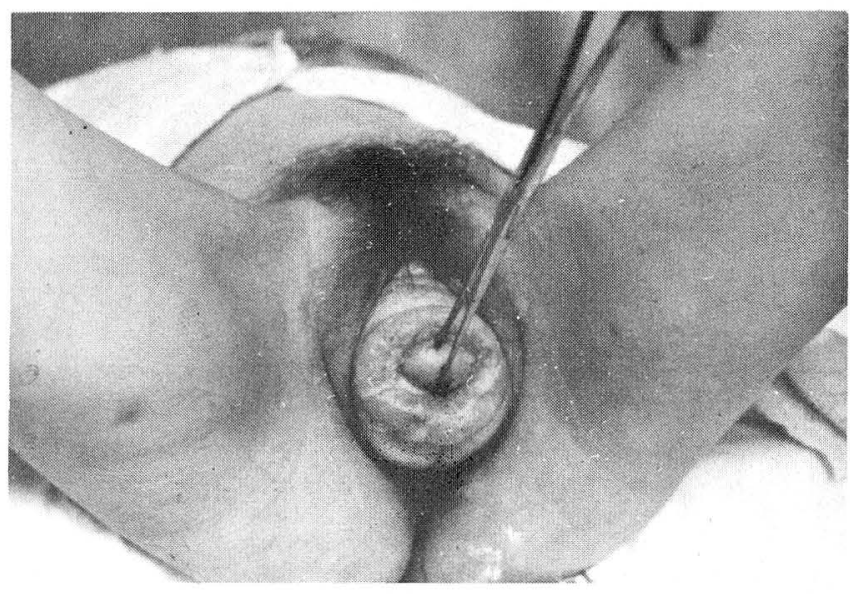

Antes de la histerectomía vaginal se demuestra que el útero desciende fácilmente.

\section{BIBLIOGRAFIA}

ROJAS LAURA, CANTILLO JAIME, OSORIO MESA ERNESTO, M.D., Revista Colombiana de Obstetricia y Ginecología. Enero - Febrero 1974.

CANTILLO J. y ROJAS L.: Miasis Vaginal (Dermatobia Hominis o Nuche). Primer Caso. Revista Colombiana de Obstetricia y Ginecología 22: 339, 1971.

MARKELL E.K. VOGE M. Uf stanford. Revista Parasitología Médica. 3ra. Edición. 1973.

BROWN H.W., U. of California. Parasitología Clínica. 4a. Edición, 1977.

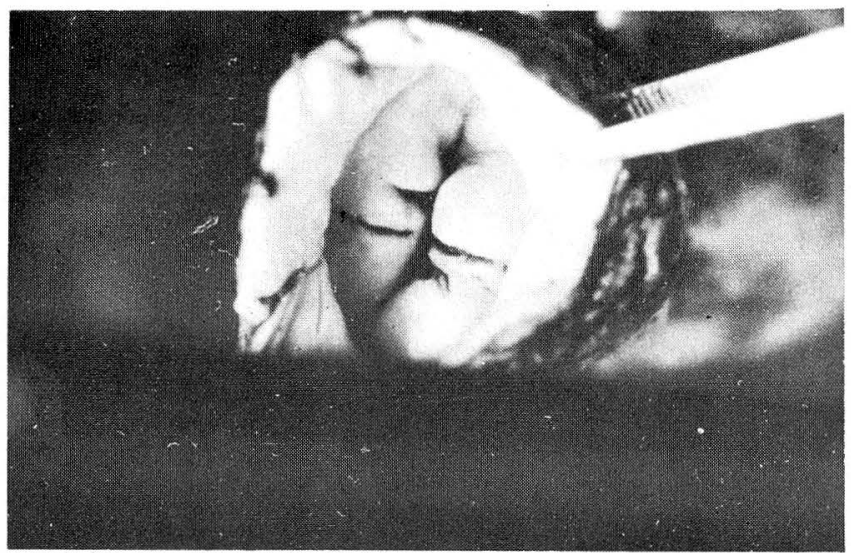

El cuello muestra las cicatrices de los túneles labrados por los gusanos

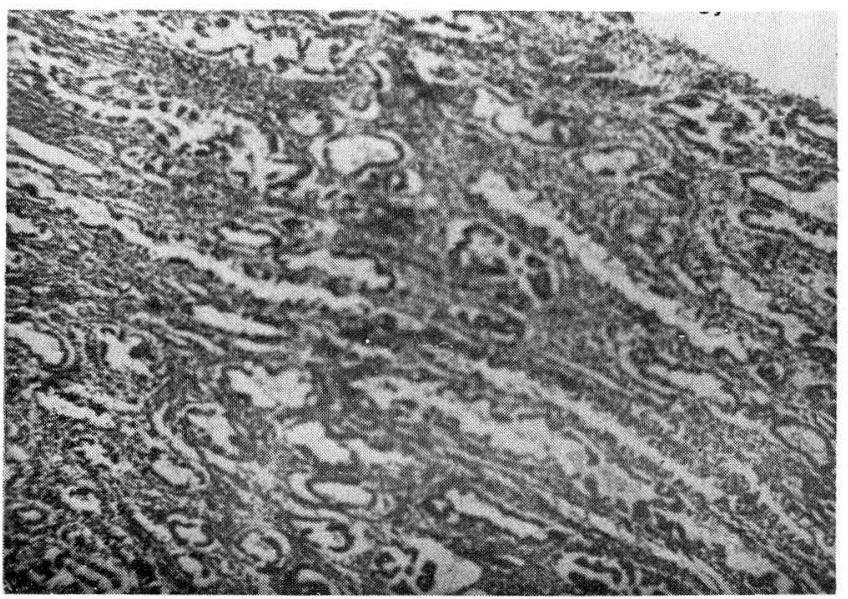

Los cortes microscópicos muestran el útero libre de gusanos

Atias A. Negheme A. Parasitología Clínica, U. de Chill, 1979.

HERMIS'S. Medical Entomdogg U. of Washington 6a. Edición, 1969.

CRAIG y FOWST. U. de Tulame N. Orleans. Parasitología Clínica. 1961.

GERARD PIEKARKI U. BONN. Tratado de Parasitología, 1959. 